\title{
Computational Model of Heat Conduction in the Steel Round Bar Bundle
}

\author{
R. WYCZÓŁKOWSKI ${ }^{a, *}$, M. GAŁA ${ }^{b}, J$. BORYCA ${ }^{a}$ \\ ${ }^{a}$ Institute of Production Management, Faculty of Production Engineering and Materials Technology, \\ Czestochowa University of Technology, Al. Armii Krajowej 19, 42-200 Czestochowa, Poland; \\ ${ }^{b}$ Institute of Electric Power Engineering, Faculty of Electrical Engineering, Czestochowa University of Technology, \\ Al. Armii Krajowej 21, 42-200 Czestochowa, Poland
}

(Received August 26, 2019; in final form September 19, 2019)

This paper presents a computational model used for quantitative analysis of heat conduction process in a flat bed of steel round bars. For this purpose, an approach based on using the analogical mathematical description of the electricity and heat flow phenomena was applied. Furthermore, this model is semi-empirical since it uses dependencies determined based on experimental research. The basis for deriving the mathematical dependencies is the geometric model of the system studied, defined by the so-called elementary cell. Each of the mechanisms of heat conduction that occurs within the cell was assigned an appropriate thermal resistance. The total thermal resistance of a cell is a combination of parallel and serial connections of individual resistances. The calculations were made for the temperature range of $0-800^{\circ} \mathrm{C}$, for four bar diameters and two porosities

DOI: 10.12693/APhysPolA.136.1001

PACS/topics: heat treatment, steel bars, bar bundle, heat conduction, thermal resistance

\section{Introduction}

Round bars belong to the basic steel products. In many cases, these elements are heat treated to ensure the required mechanical and technological properties of the bars. During these operations, the bars are most often heated in the form of cylindrically shaped bundles $[1,2]$. This is a very specific type of charge because, as shown in Fig. 1, it has a porous structure. With this feature, heat transfer in the bundle is a combination of heat conduction and thermal radiation. The knowledge of the course of these processes is fundamental for prediction of the temperature distribution in the heated charge $[3,4]$. Previous publications have been devoted to the issue of thermal radiation in the area of the bar bundle [5,6], whereas this paper presents a mathematical model describing the phenomenon of heat conduction.

\section{Analysis and modelling}

Thermal conduction through the bar bundle is a combination of the following mechanisms: conduction across individual bars, conduction in free spaces filled by gas, and contact conduction between adjacent bars. An approach of assigning individual thermal resistances to each of these mechanisms can be used to analyse heat conduction. This method is based on the analogy of the phenomena of electrical and thermal conduction,

\footnotetext{
* corresponding author; e-mail: wyczolkowski.rafal@wip.pcz.pl
}

which results from the similarity of the mathematical notation of two laws: Ohm and Fourier [7]. According to Ohm's law, electrical resistance $R_{\mathrm{el}}$ is the quotient of voltage $\Delta V$ and current flow $I$

$$
R_{\mathrm{el}}=\frac{\Delta V}{I}
$$

In the case of heat flow, analogical value to $\Delta V$ is temperature difference $\Delta t$, while current $I$ corresponds to heat flux $q$. Base on this analogy, an appropriate thermal resistance $R_{t}$ can be assigned to the electrical resistance

$$
R_{t}=\frac{\Delta t}{q} \text {. }
$$

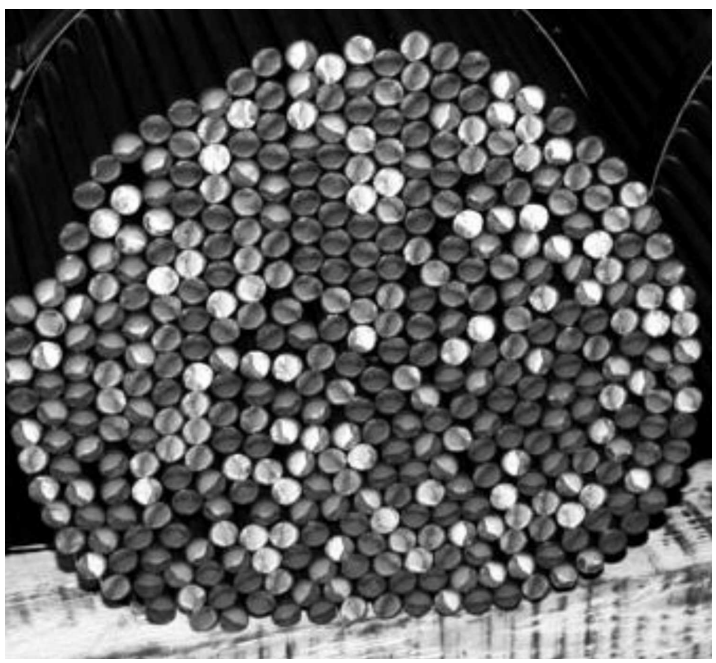

Fig. 1. The frontal surface of the round bar bundle showing its porous structure. 
(a)

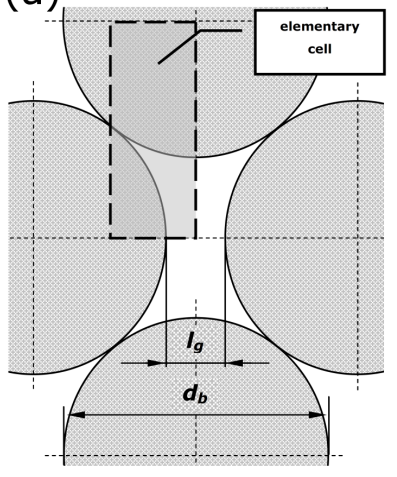

(b)

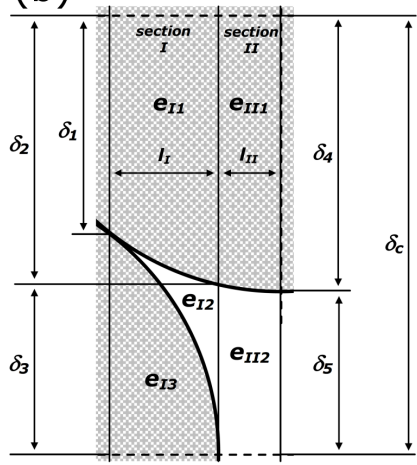

Fig. 2. Geometric model of the charge: (a) part of the bar bed, (b) elementary cell used to analyse thermal resistances.

The starting point for the analysis of the problems discussed is the geometric model of the charge. Such a model represents a flat bed of bars with a staggered packing, whose fragment is shown in Fig. 2a. The basis for establishing all mathematical relations is to isolate a repetitive part from the bed (Fig. 2b), which is termed an elementary cell. This cell contains parts of bars from two adjacent layers and is divided into two vertical sections, marked as I and II, parallel to the heat flow direction. In the boundary of section I, there are three elements $\left(e_{\mathrm{I} 1}, e_{\mathrm{I} 1}\right.$, and $\left.e_{\mathrm{I} 3}\right)$ corresponding to the part of the bar from the first layer, the part of gap and the part of the bar from the second layer, respectively. There are two elements within section II: $e_{\mathrm{II} 1}$ and $e_{\mathrm{II} 2}$, relating to the part of the bar from the top layer and the remaining part of the gap.

The geometric quantities to be used when defining the elementary cell are: the diameter of the bar $d_{b}$ and the width of the gap between the bars from one layer $l_{g}$. Based on these two parameters, the vertical dimension of the cell $\delta_{c}$ and the horizontal dimension of the sections I and II are designated

$$
\begin{aligned}
& \delta_{c}=\sqrt{d_{b}^{2}-\left[0.5\left(d_{b}+l_{g}\right)\right]^{2}}, \\
& l_{\mathrm{I}}=0.25\left(d_{b}-l_{g}\right), \\
& l_{\text {II }}=0.5 l_{g} .
\end{aligned}
$$

When analysing the thermal resistance of individual elements of the elementary cell, their dimensions in the direction of heat flow are important parameters (in Fig. 2b, these are vertical dimensions). The minimum and maximum dimensions of the individual components are presented in Table I. The values of dimensions $\delta_{1}$ and $\delta_{2}$ result from the Pythagorean theorem, because they concern one of the legs of a right triangle, the other leg with the dimensions $l_{\mathrm{I} 1}+l_{\mathrm{II}}$ and $l_{\mathrm{II}}$, whereas hypotenuse is the half of the bar diameter. The individual elements can be assigned the corresponding thermal resistances, which are also presented in Table I.
TABLE I

Minimum and maximum dimensions and thermal resistances of individual elements of the elementary cell.

\begin{tabular}{c|c|c|c}
\hline \hline Element & $\begin{array}{c}\text { Minimal } \\
\text { dimension }\end{array}$ & $\begin{array}{c}\text { Maximal } \\
\text { dimension }\end{array}$ & $\begin{array}{c}\text { Thermal } \\
\text { resistance }\end{array}$ \\
\hline$e_{\mathrm{I} 1}$ & $\delta_{1}$ & $\delta_{2}$ & $R_{\mathrm{I} 1}$ \\
$e_{\mathrm{I} 2}$ & 0 & $\delta_{3}=\delta_{c}-\delta_{2}$ & $R_{\mathrm{I} 2}$ \\
$e_{\mathrm{I} 3}$ & 0 & $\delta_{c}-\delta_{1}$ & $R_{\mathrm{I} 3}$ \\
$e_{\mathrm{II} 1}$ & $\delta_{2}$ & $\delta_{4}=0.5 d_{b}$ & $R_{\mathrm{II} 1}$ \\
$e_{\mathrm{I} 2}$ & $\delta_{5}=\delta_{c}-\delta_{4}$ & $\delta_{3}=\delta_{c}-\delta_{2}$ & $R_{\mathrm{II} 2}$
\end{tabular}
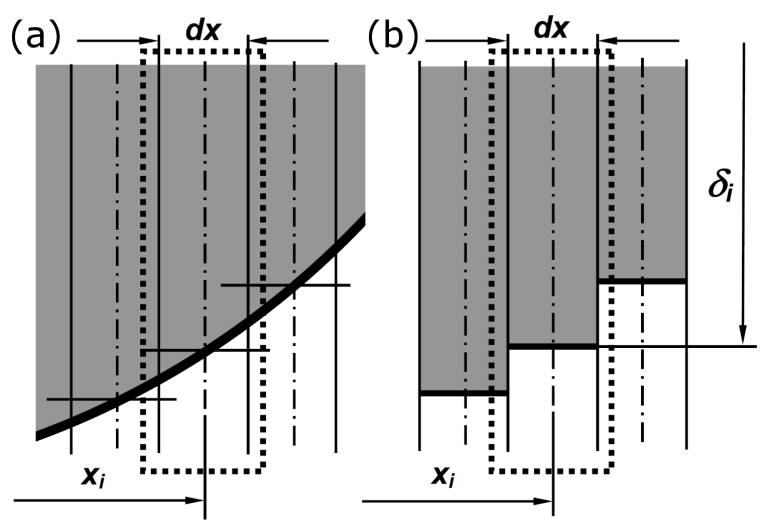

Fig. 3. The method of transforming the bar surface: (a) cylindrical surface, (b) stepped surface.

Thermal resistance $R$ of a flat layer with thermal conductivity $k$, and the dimension $\delta$ is described [7] by the equation:

$$
\mathrm{d} x=0.5 d_{b} / n .
$$

However, it cannot be applied directly to our case, because the surfaces of the individual elements are not perpendicular to the direction of heat flow. Therefore, the actual cylindrical surfaces should be replaced by stepped surfaces, as illustrated in Fig. 3. Then, the elementary cell element is divided into a series of sections parallel to the direction of heat flow with the width $\mathrm{d} x$, where $n$ is the number of sections into which half the diameter of the bar is divided.

According to the Pythagorean theorem, the dimension $\delta_{i}$ of a given section is determined from the equation

$$
\delta_{i}=\sqrt{\left(0.5 d_{b}\right)^{2}-x_{i}^{2}}
$$

where

$$
x_{i}=\mathrm{d} x(i-0.5)
$$

and $i$ is a number of the $i$-th section.

According to this methodology, the thermal resistance of the $i$-th section is

$$
R_{i}=\frac{n \delta_{i}}{k} .
$$

Thermal resistance of the entire element $R_{e}$ is calculated as a parallel connection of the resistances of individual sections. 
TABLE II

Thermal resistance of the bar with a diameter of $20 \mathrm{~mm}$ depending on the value of $n$.

\begin{tabular}{c|c|c}
\hline \hline$n$ & $\begin{array}{c}\text { Thermal resistance } \\
\times 10^{-7}\left[\mathrm{~m}^{2} \mathrm{~K} / \mathrm{W}\right]\end{array}$ & $\begin{array}{c}\text { Result } \\
\text { difference [\%] }\end{array}$ \\
\hline 10 & 2786 & 9.25 \\
50 & 2648 & 3.84 \\
100 & 2618 & 2.67 \\
500 & 2578 & 1.10 \\
1000 & 2567 & 0.67 \\
5000 & 2556 & 0.24 \\
10000 & 2553 & 0.12 \\
50000 & 2550 & 0
\end{tabular}

The value of thermal resistance of individual elements determined by means of the presented methodology, due to the transformation of cylindrical surfaces, depends on the sections number $n$. The thermal resistance of each element is more similar to the actual value, for greater values of $n$. Table II shows the influence of the value of $n$ on the results of calculation of the thermal resistance of the bar with a diameter of $20 \mathrm{~mm}$. The calculation assumes that the thermal conductivity of the bar is $50 \mathrm{~W} /(\mathrm{m} \mathrm{K})$. In the right column of Table II the percentage difference of the resistance of the $R_{b-n}$ bar obtained for particular values of $n$ relative to the resistance value $R_{b-n_{\max }}$ obtained for $n_{\max }=50000$ is presented

$$
\delta R_{b}=\frac{R_{b-n}-R_{b-n_{\max }}}{R_{b-n_{\max }}} .
$$

As can be seen in the division of the bar for 1000 sections, the difference in the result does not exceed one percent.

In calculations, the $n$-value for which the area of the elementary cell was divided was assumed to be 10000 .

In the cell area, in addition to the resistances listed in Table I, there is also the contact thermal resistance $R_{c t}$ between adjacent bars. The value of this resistance, depending on the diameter of the bar and the temperature, is calculated from the equations determined experimentally [8]:

$$
R_{c t}=\left(B_{1} t^{2}-B_{2} t+B_{3}\right) \times 10^{-4}
$$

where

$$
\begin{aligned}
& B_{1}=0.0023 d_{b}+5 \times 10^{-5}, \\
& B_{2}=-1.96 d_{b}-0.036, \\
& B_{3}=1346.5 d_{b}+47.8 .
\end{aligned}
$$

The changes in $R_{c t}$ resistance values of bar bundles with diameters of $10,20,30$, and $40 \mathrm{~mm}$ in the temperature range of $0-800^{\circ} \mathrm{C}$ are shown in Fig. 4. The smaller bar diameter is, the lower is the resistance. At the same time, this parameter assumes a minimum value for each case at a temperature of approximately $350^{\circ} \mathrm{C}$. The obtained values of resistance $R_{c t}$ are generally within the range of $5-10 \times 10^{-3} \mathrm{~m}^{2} \mathrm{~K} \mathrm{~W}^{-1}$.

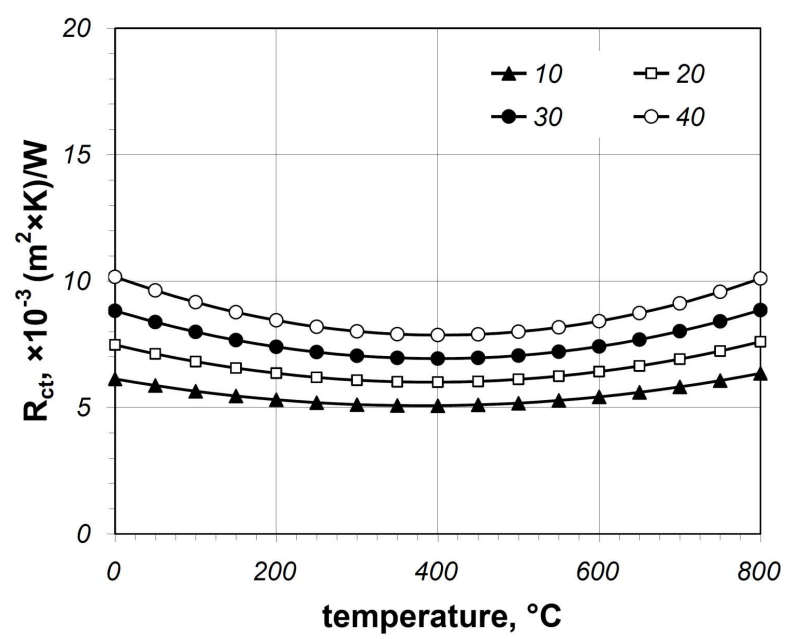

Fig. 4. Contact thermal resistance depending on the temperature and bar diameter.

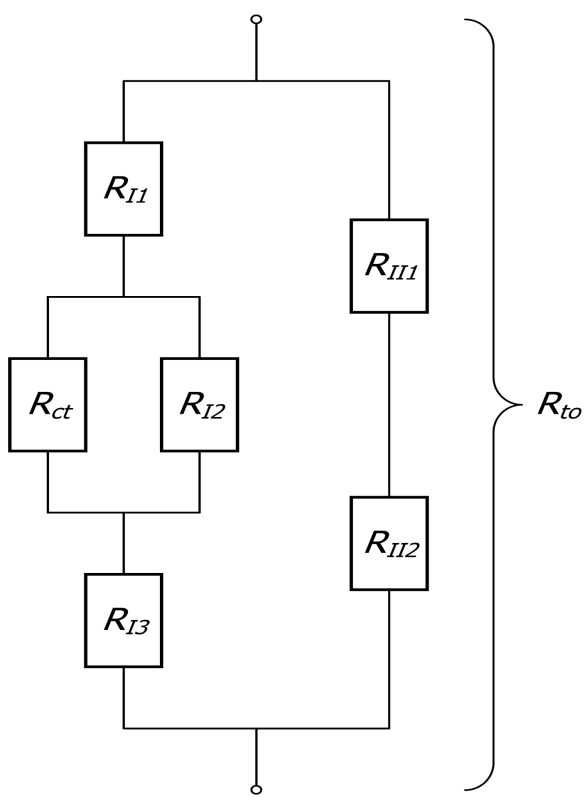

Fig. 5. Thermal resistance network for heat conduction through an elementary cell.

The total thermal resistance for a cell $R_{t o}$ is calculated as a combination of parallel and serial connections of the resistances mentioned above. The equivalent system of the connections of these resistances is shown in Fig. 5.

\section{Results and discussion}

Four bar diameters $(10,20,30$, and $40 \mathrm{~mm})$ and two porosities were taken into account in the calculations of the beds analysed. Bed porosity in the adopted geometric model is a function of the gap width $l_{g}$. Two values of this parameter were assumed for the calculations: 

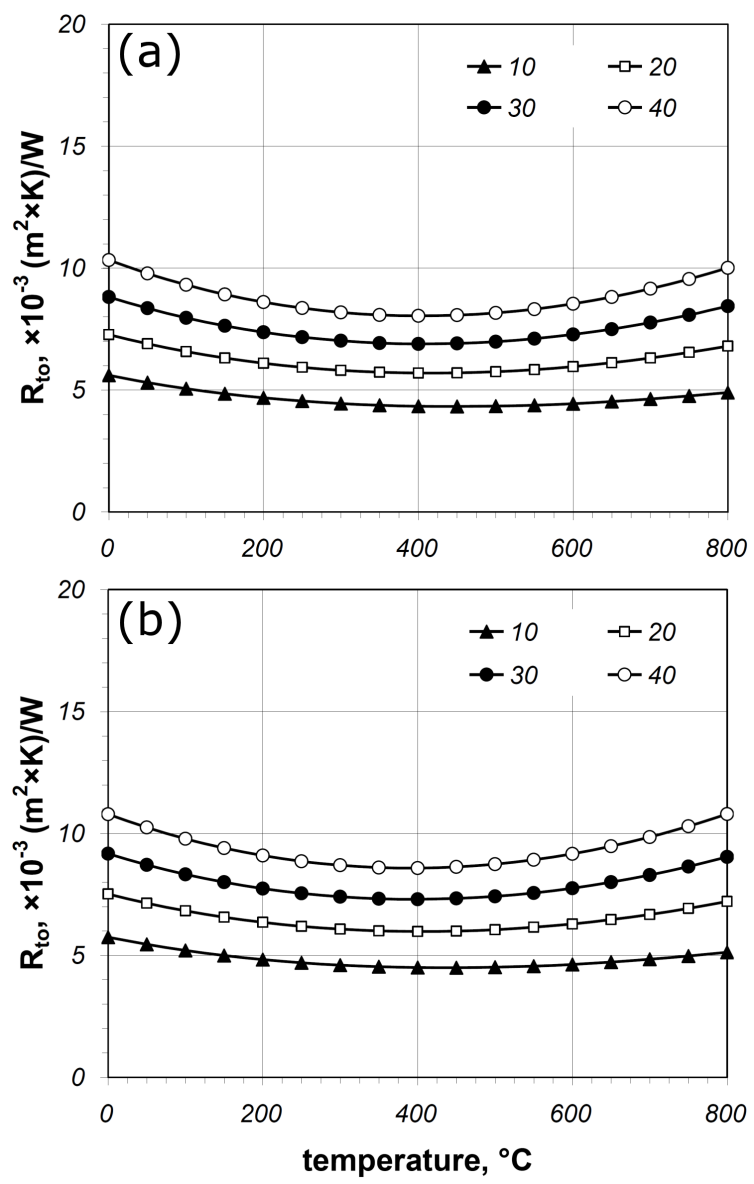

Fig. 6. Total thermal resistance of bar bed with: (a) porosity of 0.145 , (b) porosity of 0.214 .

$0.1 d_{p}$ and $0.4 d_{p}$. These values of $l_{g}$ correspond to the porosities of 0.145 and 0.214 , respectively. The model also takes into account that the bar thermal conductivity and the gas thermal conductivity change with temperature. All calculations were performed for the temperature range of $200-800^{\circ} \mathrm{C}$.

During the analysis of calculated results, the values of $R_{t o}$ resistance were presented as first (Fig. 6). Figure 6 a applies to bed with a porosity of 0.145 , while Fig. $6 \mathrm{~b}$ refers to bed with a porosity of 0.214 . The resistance $R_{t o}$ is slightly lower for beds with higher porosity. This can be explained by the fact that the dimension of the cell $\delta_{c}$ decreases with the increase of the gap between the bars, and according to the Eq. (6), the higher the value of $\delta_{2}$, the higher the thermal resistance of the element. The obtained curves, both in terms of shape and values, are very similar to curves obtained for resistance $R_{c t}$. This shows that the intensity of heat conduction in the system studied is primarily determined by the contact conductivity at the points of contact of the bars from the subsequent layers.

It is critical for the analysis to what extent the total thermal resistance of the beds analysed differs from the thermal resistance for conduction in solid steel $R_{c d}$.
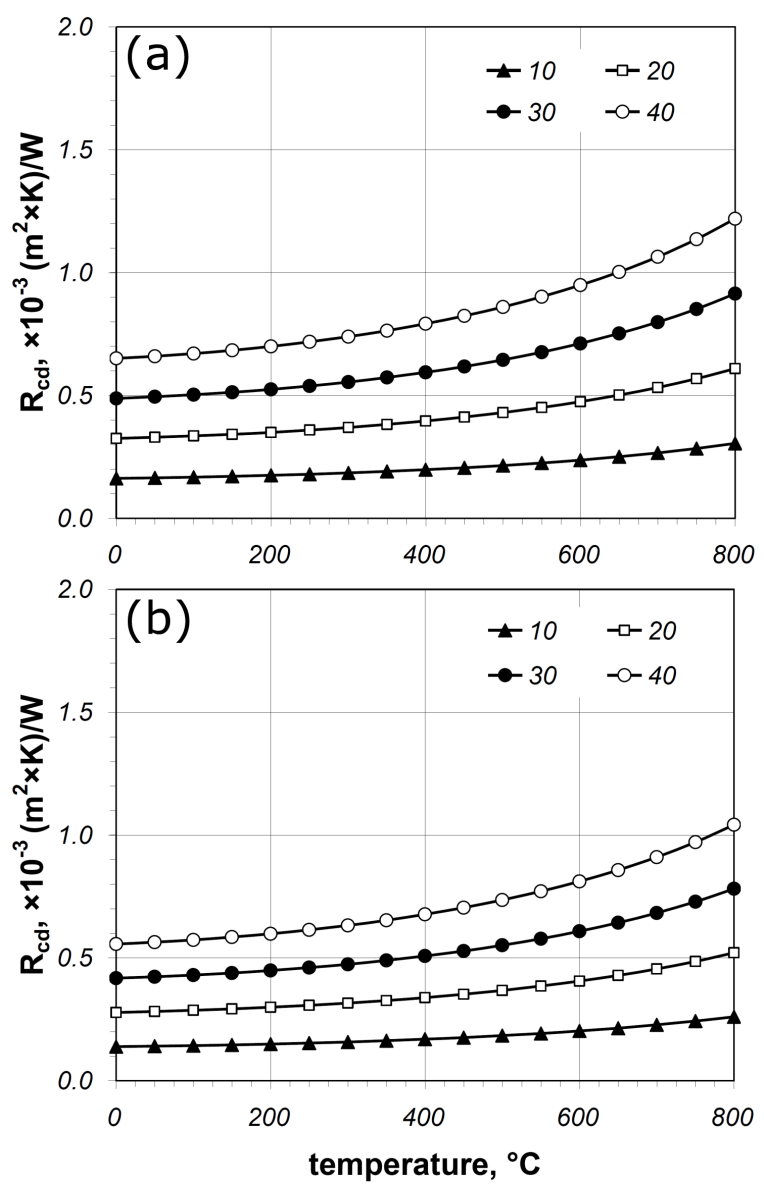

Fig. 7. Thermal resistance for conduction in solid steel: (a) results for cell C1, (b) results for cell C2.

The values of resistance $R_{c d}$ were calculated from Eq. (6), where the cell height for a given case was used in the nominator as described in the Eq. (3). Dimensions $\delta_{c}$ obtained for a bed with a porosity of 0.145 were denoted as $\mathrm{C} 1$ cell, whereas for a bed with a porosity of 0.214 - as C2. The results of calculations of resistance $R_{c d}$ for both cells depending on temperature and bar diameter are shown in Fig. 7.

As can be seen, the values of resistance $R_{c d}$, which for the cases analysed are in the range of $0.1-1.2 \times 10^{-3} \mathrm{~m}^{2} \mathrm{~K} / \mathrm{W}$, are more than an order of magnitude lower than the values of resistance $R_{s t o}$. Furthermore, the resistance $R_{c d}$ increases with temperature, which is due to the nature of the changes in the thermal conductivity of steel. A direct comparison of these two resistances was made, for which the concept of reduced resistance $R_{r d}$ defined as the quotient of $R_{t o}$ and $R_{c d}$ resistances was used

$$
R_{r d}=\frac{R_{t o}}{R_{c d}}
$$

The values of resistance $R_{r d}$ are shown in Fig. 8. It can be seen that this parameter decreases as a function of temperature and bar diameter, but increases as a function of porosity: for the porosity of 0.145 , it assumes values 

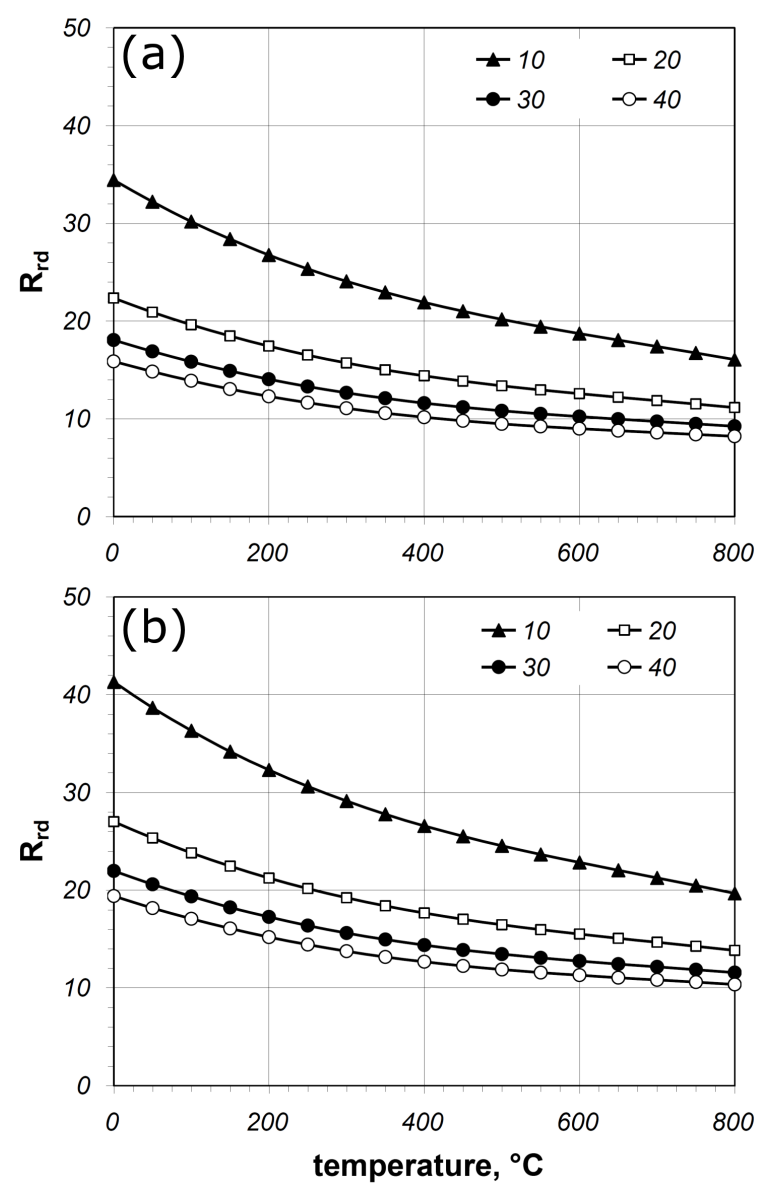

Fig. 8. Reduced thermal resistance of bed with: (a) porosity of 0.145 , (b) porosity of 0.214 .

in the range of $8-34$, whereas for the porosity of 0.214 , it is in the range of 10-42. These results show to what extent the heat conduction in a heat treated bundle of bars differs from that in solid steel. This fact makes it very difficult to choose heating curves for this type of charge.

The results presented so far show that the phenomenon of contact conduction is of key importance to the heating of the bar bundle. The intensity of this heat transfer mechanism is a function of a number of different factors and can be controlled to some extent $[9,10]$. Therefore, the resistance $R_{t o}$ was calculated for two constant values of the resistance $R_{c t}$, minimum and maximum values for curves from Fig. $4 R_{c t-\min }=5 \times 10^{-3} \mathrm{~m}^{2} \mathrm{~K} \mathrm{~W}^{-1}$, $R_{c t-\max }=10 \times 10^{-3} \mathrm{~m}^{2} \mathrm{~K} \mathrm{~W}^{-1}$.

The results of the resistance $R_{t o}$ for a bed with a porosity of 0.145 are shown in Fig. 9. It is noticeable that with a reduction in the resistance $R_{c t}$ by half, the total resistance $R_{t o}$ decreases almost proportionally. For $R_{c t-\min }$, total resistance $R_{t o}$ is in the range of $7-10 \times 10^{-3} \mathrm{~m}^{2} \mathrm{~K} \mathrm{~W}^{-1}$, whereas for $R_{c t-\max }$, this is the range of $9-19 \times 10^{-3} \mathrm{~m}^{2} \mathrm{~K} \mathrm{~W}^{-1}$.

As before, the reduced resistance $R_{r d}$ was calculated also for this case. The values of this parameter are shown
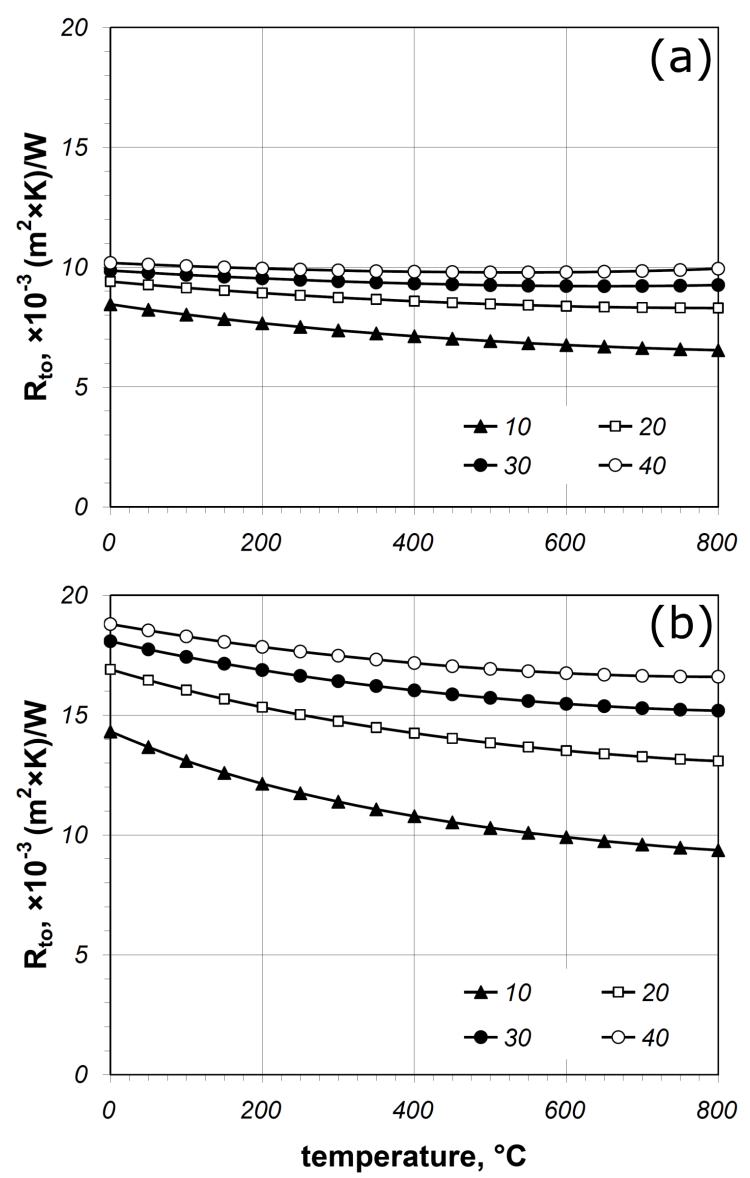

Fig. 9. Total thermal resistance of bed with a porosity of 0.145 for: (a) minimal $R_{c t}$ value, (b) maximal $R_{c t}$ value.

in Fig. 10. For $R_{c t-\min }$, the parameter $R_{r d}$ is in the range of 8-50, whereas for $R_{c t-\max }$, this range is $12-88$.

The results presented in Figs. 9 and 10 show that for heat treatment of bar bundles, the preparation of the charge should aim to ensure the best conditions possible for contact heat conduction. This will shorten the process time and help reduce energy consumption.

In the calculations presented so far, air was taken as the gas phase that fills the gaps of the charge. However, air is a gas with a relatively low thermal conductivity. In the thermal treatment of steel products such as strips and sheets, hydrogen is used to increase the intensity of the heating process [11]. This factor is characterized by the highest thermal conductivity among all gases, which in the temperature range of $0-800^{\circ} \mathrm{C}$ grows linearly from about 0.18 to $0.52 \mathrm{~W} /(\mathrm{m} \mathrm{K})[7]$.

Therefore, further calculations were made assuming that the gaseous phase of the bar bed is hydrogen. The results of these calculations for a bed with a porosity of 0.145 are shown in Fig. 11. Figure 11a shows the values of total resistance. Compared to the previous case, where the gas phase was air, the total thermal resistance is lower by approximately $2.5 \times 10^{-3} \mathrm{~m}^{2} \mathrm{~K} \mathrm{~W}^{-1}$. 

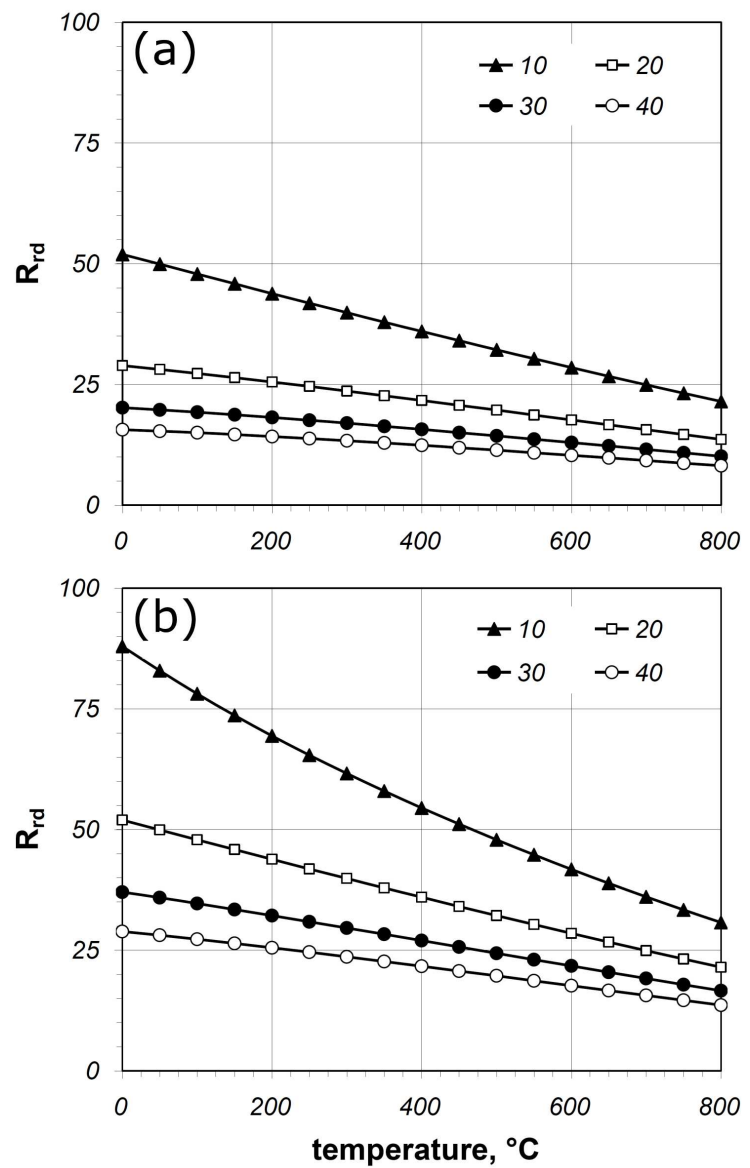

Fig. 10. Reduced thermal resistance of bed with porosity of 0.145 for: (a) minimal $R_{c t}$ value, (b) maximal $R_{c t}$ value.

In percentage terms, this is a decline in $R_{t o}$ by ca. $24 \%$. Furthermore, Fig. 11b shows the values of reduced resistance $R_{r d}$. In this case, this parameter is between 2.5 and 20. For the same bed, if air is the gas (Fig. 8a), the reduced resistance ranges from 8 to 34 . The disproportion of the $R_{r d}$ values between the two cases is particularly noticeable in the initial temperature range of $0-200{ }^{\circ} \mathrm{C}$. Therefore, if this is only possible for technological reasons, the heating of the bars in the bundles should be performed in the hydrogen atmosphere. This gas, due to its high thermal conductivity, contributes significantly to the intensification of heat exchange.

\section{Summary and conclusions}

The presented results show that the process of heat conduction in a bundle of bars, which is a porous medium, is much less intense than in solid steel. The critical factor for this process is the contact heat conduction. The thermal resistance of the analysed charge can also be reduced by changing the atmosphere in which the heat treatment is performed (replacing air with hydrogen).
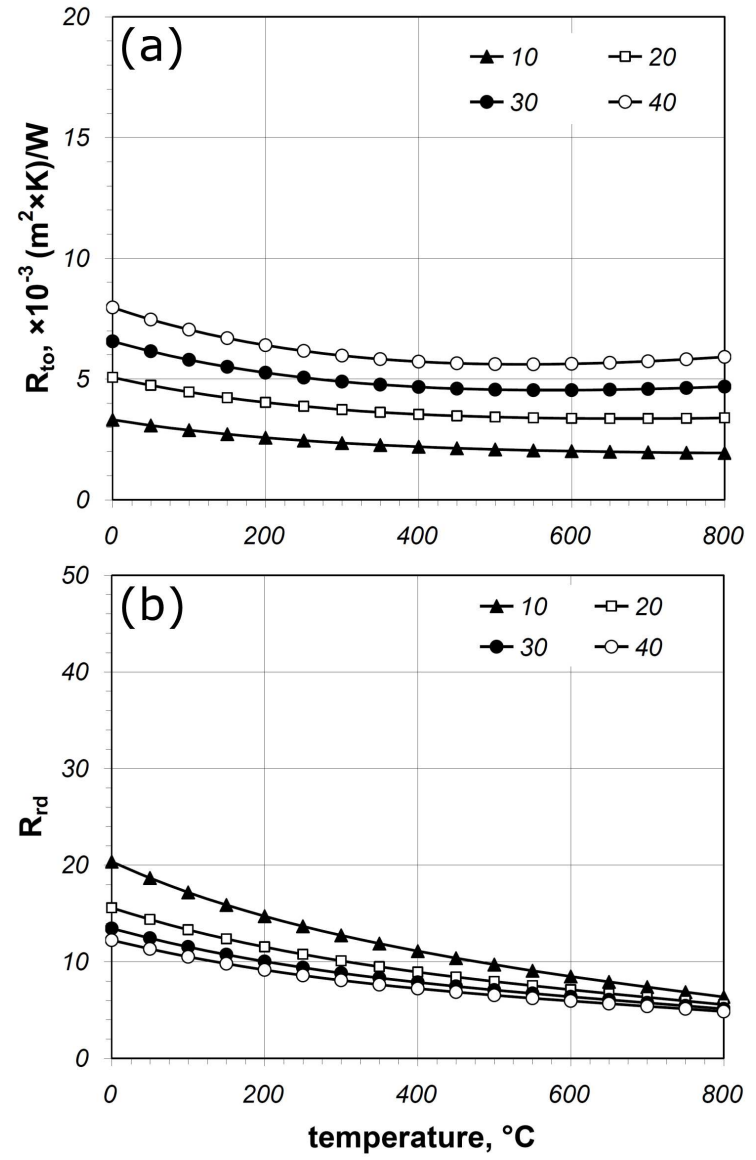

Fig. 11. Resistance of bed with porosity of 0.145 with hydrogen as gas phase: (a) total values, (b) reduced values.

The heating pattern of a bar bundle is also affected by bar diameter: the larger the diameter, the lager the resistance $R_{t o}$. On the other hand, the second geometric parameter of the charge, i.e., porosity, has no significant effect on the value of $R_{t o}$.

In conclusion, it should also be mentioned that the approach used in the mathematical model to describe heat conduction using an analogy to electricity is very convenient to use. Assigning the appropriate thermal resistance to each of the identified heat conduction mechanisms allows for building a universal and at the same time relatively simple computational model. The presented model is semi-empirical because the results of experimental studies were used to calculate the contact conduction resistance.

\section{References}

[1] S.S. Sahay, K. Krishman, Iron Steelmak. 34, 89 (2007).

[2] C. Kolmasiak, T. Wyleciał, Metalurgija 57, 368 (2018). 
[3] T.R. Rao, G.J. Barth, J.R. Miller, Iron Steeleng. 60, 22 (1983).

[4] S.S. Sahay, P.C. Kapur, Iron Steelmak. 34, 262 (2007).

[5] R. Wyczolkowski, D. Urbaniak, J. Thermophys. Heat Transf. 30, 721 (2016).

[6] R. Wyczolkowski, J. Boryca. Acta Phys. Pol. A $\mathbf{1 3 5}$ 256 (2018).

[7] Y.A. Cengel, Heat and mass transfer - A practical approach, 3rd ed., McGraw-Hill, New York 2007.
[8] R. Wyczolkowski, "Modelling of effective thermal conductivity of the steel porous charge using the thermal resistance concept", Series Monographs, Vol. 67, Częstochowa University of Technology, Częstochowa 2017 (in Polish).

[9] M. Rywotycki et al, Arch. Metallurg. 61, 2061 (2016).

[10] M.M. Yovanovitch, IEEE Transactions 20, 182 (2005).

[11] X. Zhang, F. Yu, W. Wu, Y. Zuo, Int. Jour. Thermophys 24, 395 (2003). 